\title{
Commentary Why Do We Need to Use Animal Models to Study Cognition and Aging?
}

\author{
Ann-Charlotte Granholm*,' \\ 'Department of Neurosciences, Medical University of South Carolina, Charleston, SC, USA
}

Neuropsychopharmacology (2010) 35, I62I-1622; doi: I0.1038/npp.2010.45

The article in Neuropsychopharmacology by Solas et al (2010) describes the effects of early stress caused by maternal separation upon cognitive function in adult and aged (18-month-old) rats. The authors argue that the findings may represent proof-of-concept for the use of glucocorticoid or insulin-related drugs in Alzheimer's disease (AD). They used a battery of well-established behavioral tasks, including locomotor behavior, forced swim task (depression test), object recognition task, and Morris water maze in rats. The investigators suggested that neonatal stress may increase age-related cognitive decline and therefore, they argued, it is possible that neonatal exposure to glucocorticoids may have long-term consequences for the rate and degree of cognitive impairment occurring with aging. This would then suggest that drugs that counteract stress hormones may be implicated in the prevention or treatment of severe age-related cognitive disorders such as AD.

Cognitive learning in humans is defined as the process of learning by using reason, intuition, and perception. The disadvantage of studying the effects of a specific factor in humans is that cognitive performance in humans is influenced by many factors such as culture, upbringing, education, diet habits, amount of exercise and other activities, as well as motivation and ambition. 'The dynamic polygon hypothesis' for human aging has recently been formulated to describe this cluster of genetic and environmental factors responsible for varying degrees of age-related dementia (see, eg, Fotuhi et al, 2009). The authors proposed that a balance of positive and negative factors can affect the brain throughout the lifespan to determine cognitive performance late in life. This is in line with the findings of Solas et al, and suggests that parallel lines can be drawn between behavioral outcome in rodent and human models for aging, as similar stressors appear to have long-term effects in both species.

*Correspondence: Dr A-C Granholm, Department of Neurosciences, Medical University of South Carolina, 19 Hagood Blvd., Charleston, SC 29425, USA, Tel: + I 843297 0652, Fax: + I 8437920659 ,

E-mail: Granholm@musc.edu, http//:www.musc.edu/aging

Received 4 March 2010; accepted 4 March 2010
Fotuhi et al (2009) suggested that a multitude of internal (genetic) and external (environmental) factors increase or decrease the cerebral blood flow, pro- and anti-inflammatory cytokines, insulin-signaling components, growth factors, cortisol, etc., leading to a congruence of physiological processes in the brain making up human learning and memory parameters. As most of the external factors can be controlled in animal models, we often use rodent models for examining the cognitive effects of certain drugs or specific external factors under controlled conditions. In rodents, it is possible to remove one factor at a time to explore how it influences the structural or functional components of the limbic system.

The investigators found that neonatal stress gave rise to long-lasting effects on amyloid precursor protein (APP) processing, favoring the amyloidogenic (damaging) type of splicing. To fully explore the role of amyloid in AD, genetic manipulations have been undertaken in mice. Work from other investigators using these transgenic mutated APP models showed that restraint stress gave rise to increased amyloid pathology in AD transgenic mice (Lee et al, 2009), but similar findings have not been reported previously for intact aged rats. As summarized by Dong and Csernansky (2009), application of stress hormones can also affect the accumulation of amyloid in transgenic $\mathrm{AD}$ models, further substantiating the findings by Solas et al in the current issue. Even though the investigators in the present article did not utilize $\mathrm{AD}$ transgenic mouse models, they utilized a well-characterized rat strain for these studies. Wistar rats have been used previously in aging studies, and manifest alterations in learning and memory processes around the age utilized in the present study (18 months of age; see Assunção et al, 2010).

Another major factor in favor of rodent models of agerelated memory decline is the lifespan. While humans live for around eight decades, and do not manifest significant rates of memory decline until their fifth or sixth decade, the most commonly used rat strains for aging studies exhibit memory loss at 18-20 months of age and significant decline at 24 months of age (Bimonte et al, 2003; BimonteNelson et al, 2008). Naturally, it is easier to perform 
longitudinal or cross-sequential studies of memory decline with aging in rats than in humans because of the difference in lifespan. Aging is a complex process that does not occur in a linear fashion, and that can be significantly different even in individuals from the same strain. Based on the complexity of the systems involved in cognitive function, the study of cognitive impairment with aging does not lend itself well to invertebrate models or computational neuronal modeling.

In the present study, the investigators chose to focus on male offspring to study the effects of maternal separation during aging. Many studies have shown that male vs female rats respond differently to stress (see, eg, Darnaudéry and Maccari, 2008). Previous work has shown the effects of a strong interaction between estradiol and glucocorticoid hormones on cognition. Therefore, it is important to consider gender as well as hormonal status when examining stress-related effects on memory in any species. Many investigators have chosen to focus their aging studies on male rats to avoid the influence of estradiol on memory performance, even though very interesting sex differences can be noted when comparing male and female rats, as demonstrated in a previous study from our group (Bimonte-Nelson et al, 2008). Because male and female rats age at a different rate, and are influenced at least partially by different factors, it is important to include several different age groups (or, alternatively, perform longitudinal behavioral testing in the same rats) to fully elucidate the aging process.

\section{CONCLUSIONS}

There is an ongoing discussion regarding what represents the best model system to study cognitive impairment with aging. It is of course true that most animal models of aging have validity, when taken into their right context, and when considering their specific conditions. Different model systems will model different aspects of cognitive impairment, from studies in Caenorhabditis elegans to rodents to humans, and it is only when we integrate these ideas from several perspectives that we can finally get a clear picture of which factors contribute to dementia or other forms of age-related memory loss.

\section{DISCLOSURE}

The author declares that she has received no contract research funding during the last three years from the industry and has no patent applications at the time of submitting this commentary.

\section{REFERENCES}

Assunção M, Santos-Marques MJ, Carvalho F, Andrade JP (2010). Green tea averts age-dependent decline of hippocampal signaling systems related to antioxidant defenses and survival. Free Radic Biol Med 48: 831-838.

Bimonte HA, Nelson ME, Granholm AC (2003). Age-related deficits as working memory load increases: relationships with growth factors. Neurobiol Aging 24: 37-48.

Bimonte-Nelson HA, Granholm AC, Nelson ME, Moore AB (2008). Patterns of neurotrophin protein levels in male and female Fischer 344 rats from adulthood to senescence: how young is 'young' and how old is 'old'? Exp Aging Res 34: 13-26.

Darnaudéry M, Maccari S (2008). Epigenetic programming of the stress response in male and female rats by prenatal restraint stress. Brain Res Rev 57: 571-585.

Dong H, Csernansky JG (2009). Effects of stress and stress hormones on amyloid-beta protein and plaque deposition. J Alzheimer's Dis 18: 459-469.

Fotuhi M, Hachinski V, Whitehouse PJ (2009). Changing perspectives regarding late-life dementia. Nat Rev Neurol 5: 649-658.

Lee KW, Kim JB, Seo JS, Kim TK, Im JY, Baek IS et al (2009). Behavioral stress accelerates plaque pathogenesis in the brain of Tg2576 mice via generation of metabolic oxidative stress. J Neurochem 108: 165-175.

Solas M, Aisa B, Mugueta MC, Del Río J, Tordera R, Ramírez MJ (2010). Interactions between age, stress and insulin on cognition: implications for Alzheimer's disease. Neuropsychopharmacology 35: $1664-1673$. 